\title{
Paramagnetic colloidal ribbons in a precessing magnetic field
}

\author{
R. Alvarez-Nodarse,${ }^{1}$ N. R. Quintero, ${ }^{2}$ F. G. Mertens,${ }^{3}$ N. Casic,${ }^{3}$ and Th. M. Fischer ${ }^{3}$ \\ ${ }^{1}$ IMUS \& Departamento de Análisis Matemático, Universidad de Sevilla, Apartado 1160, E-41080 Sevilla, Spain \\ ${ }^{2}$ IMUS \& Departamento de Física Aplicada I, E.P.S., Universidad de Sevilla, c/ Virgen de África 7, E-41011 Sevilla, Spain \\ ${ }^{3}$ Physikalisches Institut, Universität Bayreuth, 95440 Bayreuth, Germany
}

(Received 23 October 2014; published 13 March 2015)

\begin{abstract}
We investigate the dynamics of a kink in a damped parametrically driven nonlinear Klein-Gordon equation. We show by using a method of averaging that, in the high-frequency limit, the kink moves in an effective potential and is driven by an effective constant force. We demonstrate that the shape of the solitary wave can be controlled via the frequency and the eccentricity of the modulation. This is in accordance with the experimental results reported in a recent paper [Casic et al., Phys. Rev. Lett. 110, 168302 (2013)], where the dynamic self-assembly and propulsion of a ribbon formed from paramagnetic colloids in a time-dependent magnetic field has been studied.
\end{abstract}

DOI: 10.1103/PhysRevE.91.032908

PACS number(s): 05.45.Yv, 87.15.hm

\section{INTRODUCTION}

Dynamic self-assembly gives rise to complex spatiotemporal structures with dynamic response functions that transcend the behavior of its individual constituents. Peaks in the dynamic susceptibilities of such structures reveal new collective oscillatory or relaxational modes, i.e. eigenmodes of dynamic conformational collective fluctuations around its equilibrium, stationary, periodic, or quasiperiodic orbits. Dielectric spectroscopy [1,2], nuclear magnetic resonance techniques [3], and optical spectroscopy [4] all measure dynamic susceptibilities. Theoretical concepts for these response functions are then applied in order to attain insight into molecular conformational modes that cannot be directly visualized. In mesoscopic systems, one has the opportunity to simultaneously probe dynamic susceptibilities and visualize the conformational changes caused by external dynamic perturbations.

The current work derives the frequency dependence of a particular response function, namely the anisotropy $\Delta \chi(\omega)$ of the dynamic susceptibility of a ribbon of paramagnetic colloids that is subject to a dynamic external magnetic field. Details of the preparation and manipulation of the colloidal ribbon have been published elsewhere [5]. In short, the ribbon consists of two colloidal chains of paramagnetic beads of diameter $2.8 \mu \mathrm{m}$ immersed in water and prepared such that they lie side by side on a glass substrate. A constant external magnetic field along the major ribbon axis stabilizes the bonds between individual paramagnetic beads in both chains, while external transversal dynamic magnetic fields induce attractive interactions between both chains of the ribbon. The cross section of the ribbon is anisotropic due to the presence of two chains. Constant gravitational, hard-core interaction torques from the glass substrate and dynamic magnetic torques act on the cross section of the ribbon.

The ratio of inertial forces and torques on the colloids to viscous forces and torques is characterized by the Reynolds number $\mathcal{R}$. Typical Reynolds numbers in colloids are small $(\mathcal{R} \ll 1)$ and inertial effects occur for transient times that are relatively short. The magnetic field $\mathbf{H}(t)$ induces magnetic moments $\mathbf{m}(t)=\mu_{0} V \chi \cdot \mathbf{H}(t)$. Here $\mu_{0}$ denotes the permeability of the vacuum, $V$ the volume of the ribbon, and $\chi$ the effective susceptibility. The dimensionless Mason number $\mathcal{M}=\eta \Omega / \mu_{0} \chi^{2} H^{2}$ characterizes the ratio of viscous vs magnetic interactions, where $\eta=10^{-3} \mathrm{~N} \mathrm{~s} \mathrm{~m}^{-2}$ denotes the water viscosity, and $\Omega$ the modulation frequency at which the direction of the magnetic field changes. Under the conditions used here, the Mason number is large $(\mathcal{M}>1)$ and the motion of the beads has a lower rate $\Omega_{\text {motion }}<\Omega$ than that of the magnetic field because viscous forces and torques are too strong to allow for a synchronous $\left(\Omega_{\text {motion }}=\Omega\right)$ motion. In fact, a sufficiently large Mason number must be chosen to ensure the integrity of our ribbon. Frequencies $\Omega / 2 \pi>11 \mathrm{~Hz}$ are necessary to prevent disintegration of the ribbon. Above this lower bound the intraparticle dynamics is slow compared to the modulation of the external field. The separation of time scales allows us to also separate dynamic conformational variables of the particle assembly into larger slow and smaller fast components.

Our system is driven by a time-dependent magnetic field $\mathbf{H}(t)=\hat{H}_{x} \mathbf{e}_{x}+\hat{H}_{+} \mathbf{e}_{+} e^{i \Omega t}+\hat{H}_{-} \mathbf{e}_{-} e^{-i \Omega t}$ with $\mathbf{e}_{ \pm}=\mathbf{e}_{y} \pm i \mathbf{e}_{z}$ and with $\hat{H}_{x} \gg \hat{H}_{+} \gg \hat{H}_{-}$. Here the field in the $x$ direction stabilizes the backbone of the ribbon, the positive circular polarized field exerts a torque on the ribbon that tries to rotate the ribbon, and the smaller negative circular polarized field induces a positive interference in the direction of gravity and a negative interference in the horizontal cross-section direction. This help counteracts the gravitational torque. The result torques on the ribbon is travelling twist walls through the ribbon. The frequency-dependent shape of these twist walls as well as the anisotropic susceptibility of the cross section are the subject of the current theoretical calculations.

In [5], the following equation was proposed to describe the dynamics of the orientation angle field $\phi_{b}(X, \tau)$ of the cross section of the paramagnetic colloidal ribbons in a precessing magnetic field along the backbone (characterized by the coordinate $X$ ) of the ribbon as a function of the dimensionless time $\tau$,

$$
\begin{aligned}
& \frac{\partial^{2}}{\partial \tau^{2}} \phi_{b}(X, \tau)+\Gamma \frac{\partial}{\partial \tau} \phi_{b}(X, \tau)=-\frac{\delta F}{\delta \phi_{b}} \\
& F=\int d X\left[U_{\mathrm{grav}}\left(\phi_{b}\right)+\Delta \chi(2 \Omega) U_{\mathrm{magn}}\left(\phi_{b}, \tau\right)\right. \\
& \left.\quad+\frac{1}{2}\left(\frac{\partial \phi_{b}}{\partial X}\right)^{2}\right]
\end{aligned}
$$


where

$$
\begin{aligned}
U_{\text {grav }}\left(\phi_{b}\right)= & \left|\sin \phi_{b}\right|, \\
U_{\text {magn }}\left(\phi_{b}, \tau\right)= & h_{+}^{2} \cos \left(2 \phi_{b}-2 \Omega \tau\right)+h_{-}^{2} \cos \left(2 \phi_{b}+2 \Omega \tau\right) \\
& +2 h_{+} h_{-} \cos (2 \Omega \tau) .
\end{aligned}
$$

The first term in (1.1) is the inertial term, and the second term is the dissipative damping from the surrounding fluid that scales with the damping parameter $\Gamma \propto \mathcal{R}^{-1}$, i.e., it is inversely proportional to the Reynolds number. Since the Reynolds number is small $(\mathcal{R} \ll 1)$, the damping factor $\Gamma \gg 1$ is large. The parameters $h_{+}$and $h_{-}$are dimensionless amplitudes of the positive and negative circularly polarized magnetic fields that oscillate with the modulation frequency $\Omega$.

A straightforward calculation leads to the following partial differential equation (PDE):

$$
\begin{aligned}
\frac{\partial^{2} \phi_{b}}{\partial \tau^{2}} & -\frac{\partial^{2} \phi_{b}}{\partial X^{2}}+\Gamma \frac{\partial \phi_{b}}{\partial \tau}+\frac{\partial}{\partial \phi_{b}}\left|\sin \phi_{b}\right| \\
= & 2 \Delta \chi(\omega)\left[h_{+}^{2} \sin \left(2 \phi_{b}-2 \Omega \tau\right)+h_{-}^{2} \sin \left(2 \phi_{b}+2 \Omega \tau\right)\right. \\
& \left.+2 h_{+} h_{-} \sin \left(2 \phi_{b}\right)\right] .
\end{aligned}
$$

In order to solve the equation above it is convenient to rewrite it by performing the following change of variables:

$\phi(x, t)=2 \phi_{b}(X, \tau), \quad t=2 \tau, \quad x=2 X, \quad \omega=2 \Omega, \quad \gamma=\frac{\Gamma}{2}$,

thus we have the equation in a standard form,

$$
\begin{aligned}
& \frac{\partial^{2} \phi}{\partial t^{2}}-\frac{\partial^{2} \phi}{\partial x^{2}}+V^{\prime}(\phi) \\
& \quad=-\gamma \frac{\partial \phi}{\partial t}+\epsilon_{1} \sin (\phi) \cos \omega t-\epsilon_{2} \cos (\phi) \sin \omega t,
\end{aligned}
$$

where

$$
V(\phi)=\left|\sin \frac{\phi}{2}\right|-\epsilon_{3}(1-\cos \phi)
$$

and $\epsilon_{1}=\Delta \chi(\omega)\left(h_{+}^{2}+h_{-}^{2}\right), \quad \epsilon_{2}=\Delta \chi(\omega)\left(h_{+}^{2}-h_{-}^{2}\right), \quad \epsilon_{3}=$ $2 \Delta \chi(\omega) h_{+} h_{-}$. The positive circularly polarized amplitude is large compared to the negative circularly polarized amplitude and hence $\epsilon_{1} \gtrsim \epsilon_{2} \gg \epsilon_{3}$.

In the following sections a theoretical and numerical analysis of the PDE (1.3) is provided.

\section{SOLVING A DAMPED EQUATION RELATED WITH A SELF-ASSEMBLED HELICAL RIBBON}

Let us consider the following equation:

$$
\begin{aligned}
& \frac{\partial^{2} \phi}{\partial t^{2}}-\frac{\partial^{2} \phi}{\partial x^{2}}+V^{\prime}(\phi) \\
& =f-\gamma \frac{\partial \phi}{\partial t}+\epsilon_{1} \sin \phi \cos \omega t-\epsilon_{2} \cos (\phi) \sin \omega t,
\end{aligned}
$$

where

$$
V(\phi)=\alpha\left|\sin \frac{\phi}{2}\right|-\epsilon_{3}(1-\cos \phi), \quad \alpha>0 .
$$

This equation agrees with Eq. (1.3) on setting

$$
\alpha=1, \quad f=0 .
$$

We are interested in the case when the frequency $\omega$ is sufficiently large (in a sense that will be explained later on) and the values of $\epsilon_{1}$ and $\epsilon_{2}$ are not necessarily small. Here, the function $\Delta \chi(\omega)$ is assumed to be an increasing function of $\omega$ such that, for relatively low frequencies, it is practically zero. Since the potential (2.2) is not a derivable function the theory of distributions [6] is needed to obtain its derivatives (see the Appendix for more details). In particular, we have

$$
\left|\sin \left(\frac{\phi}{2}\right)\right|^{\prime}=\frac{1}{2} \operatorname{sgn}\left[\sin \left(\frac{\phi}{2}\right)\right] \cos \left(\frac{\phi}{2}\right) .
$$

\section{A. Method of averaging}

To solve Eq. (2.1), we use a method of averaging (see, e.g., [7-9]). The idea is to transform (2.1) into a new PDE with an effective potential independent of $t$ [see Eq. (2.9) below]. This method has been successfully applied to explain the following: the dynamics of the kinks in a parametrically driven discrete $\phi^{4}$ model with loss [8]; the dynamics of sineGordon kinks in the presence of rapidly varying parametric periodic perturbations [10,11]; and solitons in optical fibers described by the nonlinear Schrödinger equation with periodic amplification [11,12]; among others.

The first step of the method of averaging used here consists of splitting the solution of Eq. (2.1), $\phi(x, t)$, into two components: a slowly varying function $\Phi(x, t)$ and a fast and small varying function $\zeta(t)$ (see, e.g., [8,9]) which describes the fast oscillations around $\Phi(x, t)$ with a frequency close to $\omega$ (see Sec. $\oint 30$ in [9]). Here, we are interested in the case when the amplitudes $\epsilon_{1}$ and $\epsilon_{2}$ of the driving force are, in general, not small in comparison with the forces due to the potential $V$. We also assume that $\zeta$ is independent of $x[7,8]$ and has zero mean [9] (see also [7,8]).

Notice that the original potential is not derivable at $\Phi=$ $0,2 \pi, \ldots$, so in order to apply the standard method of averaging it is necessary to perform an additional approximation. The idea is to exchange the function $v(\phi)=\left|\sin \frac{\phi}{2}\right|$ by a smooth function $\tilde{v}$ such that $\tilde{v}^{\prime}$ admits a Taylor expansion (see the Appendix for more details). Then we can apply the standard averaging method to the new PDE with this approximate smooth potential. Since both functions $v$ and $\tilde{v}$ coincide, except in a very small neighborhood of the origin, one can expect a good agreement between the solutions of the original Eq. (2.1) and the approximate averaged equation. This last statement is checked by the simulations in Sec. IIC. For the sake of simplicity in the following we use the same letter $v$ for the approximate smooth potential.

By substituting $\phi(x, t)=\Phi(x, t)+\zeta(t)$ into (2.1) we obtain

$$
\begin{aligned}
\frac{\partial^{2} \Phi}{\partial t^{2}} & -\frac{\partial^{2} \Phi}{\partial x^{2}}+\frac{\partial^{2} \zeta}{\partial t^{2}}+V^{\prime}(\Phi+\zeta) \\
= & f-\gamma \frac{\partial \Phi}{\partial t}-\gamma \frac{\partial \zeta}{\partial t}+\epsilon_{1} \sin (\Phi+\zeta) \cos \omega t \\
& -\epsilon_{2} \cos (\Phi+\zeta) \sin \omega t .
\end{aligned}
$$


Since $\zeta$ is sufficiently small we can use the formal Taylor expansions

$$
\begin{aligned}
V^{\prime}(\Phi+\zeta) & =V^{\prime}(\Phi)+V^{\prime \prime}(\Phi) \zeta+V^{\prime \prime \prime}(\Phi) \frac{\zeta^{2}}{2}+\cdots \\
\sin (\Phi+\zeta) & =\sin (\Phi)+\cos (\Phi) \zeta+\cdots \\
\cos (\Phi+\zeta) & =\cos (\Phi)-\sin (\Phi) \zeta+\cdots
\end{aligned}
$$

By substituting the above expressions into (2.5), this becomes

$$
\begin{aligned}
\frac{\partial^{2} \Phi}{\partial t^{2}} & -\frac{\partial^{2} \Phi}{\partial x^{2}}+\frac{\partial^{2} \zeta}{\partial t^{2}}+V^{\prime}(\Phi)+V^{\prime \prime}(\Phi) \zeta+V^{\prime \prime \prime}(\Phi) \frac{\zeta^{2}}{2}+\cdots \\
= & f-\gamma \frac{\partial \Phi}{\partial t}-\gamma \frac{\partial \zeta}{\partial t}+\epsilon_{1}[\sin (\Phi)+\cos (\Phi) \zeta] \cos \omega t \\
& -\epsilon_{2}[\cos (\Phi)-\sin (\Phi) \zeta] \sin \omega t+\cdots
\end{aligned}
$$

Following [8,9] we collect, on the one hand, the fast varying terms, and on the other hand, the slowly varying terms. For the fast varying terms, we have, in the first order of $\zeta$, the equation

$$
\frac{\partial^{2} \zeta}{\partial t^{2}}+\gamma \frac{\partial \zeta}{\partial t}=\epsilon_{1} \sin (\Phi) \cos \omega t-\epsilon_{2} \cos (\Phi) \sin \omega t
$$

where $\Phi$ is assumed to be a constant (since $\zeta$ is a very fast varying function in comparison with $\Phi$ ). In Eq. (2.7), the term $V^{\prime \prime}(\Phi) \zeta$ is neglected since it is small in comparison with the other terms (this is because $\dot{\zeta} \sim \omega, \ddot{\zeta} \sim \omega^{2}$ ).

The general solution of Eq. (2.7) is

$$
\begin{aligned}
\zeta(t)= & C_{1}+C_{2} e^{-\gamma t}+\frac{\epsilon_{1} \gamma \sin \Phi+\epsilon_{2} \omega \cos \Phi}{\omega\left(\gamma^{2}+\omega^{2}\right)} \sin (\omega t) \\
& -\frac{\epsilon_{1} \omega \sin \Phi-\epsilon_{2} \gamma \cos \Phi}{\omega\left(\gamma^{2}+\omega^{2}\right)} \cos (\omega t) .
\end{aligned}
$$

The initial conditions can be chosen such that $C_{1}=0$ (recall that $\zeta$ should be a zero mean function of $t$ ), and therefore, after a transient time $\tau \gg 1 / \gamma$, we have

$$
\begin{aligned}
\zeta(t)= & \frac{\epsilon_{1} \gamma \sin \Phi+\epsilon_{2} \omega \cos \Phi}{\omega\left(\gamma^{2}+\omega^{2}\right)} \sin (\omega t) \\
& -\frac{\epsilon_{1} \omega \sin \Phi-\epsilon_{2} \gamma \cos \Phi}{\omega\left(\gamma^{2}+\omega^{2}\right)} \cos (\omega t) .
\end{aligned}
$$

From the above explicit expression of $\zeta(t)$, it is clear that the condition for $\zeta$ to be sufficiently small means that $\left|\epsilon_{1,2}\right| \ll \omega^{2}$.

Notice that, since $\langle\zeta\rangle=0$, then $\langle\phi\rangle=\langle\Phi\rangle$. Therefore, if we insert (2.8) into (2.6), take the average $\langle\cdot\rangle:=1 / T \int_{0}^{T} \cdot d t$, $T=2 \pi / \omega$, and use the relations

$$
\begin{aligned}
\left\langle\zeta^{2}\right\rangle & =\frac{\epsilon_{1}^{2} \sin ^{2}(\Phi)+\epsilon_{2}^{2} \cos ^{2}(\Phi)}{2 \omega^{2}\left(\omega^{2}+\gamma^{2}\right)} \\
\langle\zeta \cos (\omega t)\rangle & =-\frac{\epsilon_{1} \sin (\Phi) \omega-\epsilon_{2} \cos (\Phi) \gamma}{2 \omega\left(\omega^{2}+\gamma^{2}\right)} \\
\langle\zeta \sin (\omega t)\rangle & =\frac{\epsilon_{1} \sin (\Phi) \gamma+\epsilon_{2} \cos (\Phi) \omega}{2 \omega\left(\omega^{2}+\gamma^{2}\right)}
\end{aligned}
$$

then we obtain, up to the order $1 / \omega^{3}$, the following averaging equation for the slowly varying term (for the sake of simplicity we use the notation $\Phi=\langle\Phi\rangle)$ :

$$
\begin{gathered}
\frac{\partial^{2} \Phi}{\partial t^{2}}-\frac{\partial^{2} \Phi}{\partial x^{2}}+V^{\prime}(\Phi)+\frac{\epsilon_{1}^{2}-\epsilon_{2}^{2}}{4\left(\omega^{2}+\gamma^{2}\right)} \sin (2 \Phi) \\
=f+\frac{\gamma \epsilon_{1} \epsilon_{2}}{2 \omega\left(\omega^{2}+\gamma^{2}\right)}-\gamma \frac{\partial \Phi}{\partial t} .
\end{gathered}
$$

Thus, we obtain the effective equation

$$
\frac{\partial^{2} \Phi}{\partial t^{2}}-\frac{\partial^{2} \Phi}{\partial x^{2}}+\frac{d V_{\mathrm{eff}}(\Phi)}{d \Phi}=f_{\mathrm{eff}}-\gamma \frac{\partial \Phi}{\partial t},
$$

where we have, respectively, the following effective potential and driving force:

$$
\begin{aligned}
V_{\mathrm{eff}}(\Phi)= & \alpha\left|\sin \frac{\Phi}{2}\right|-\epsilon_{3}(1-\cos \Phi) \\
& +\frac{\epsilon_{1}^{2}-\epsilon_{2}^{2}}{8\left(\gamma^{2}+\omega^{2}\right)}(1-\cos 2 \Phi), \\
f_{\mathrm{eff}}= & f+\frac{\gamma \epsilon_{1} \epsilon_{2}}{2 \omega\left(\gamma^{2}+\omega^{2}\right)} .
\end{aligned}
$$

The approximate equation above only holds if the frequency of the driving force is sufficiently large (larger than the internal frequencies of the unperturbed system).

Let us analyze the shape of the kinks predicted by Eq. (2.9) by examining the effective potential (2.10). We follow the idea described in [13], i.e., we find the minima of (2.10) since these determine the shape of the kink solutions of Eq. (2.1) (see [13] for further details). We assume that all the parameters are positive real numbers, i.e.,

$$
\alpha>0, \quad \epsilon_{1}>0, \quad \epsilon_{2}>0, \quad \epsilon_{3}>0, \text { and } \epsilon_{1}>\epsilon_{2} .
$$

We recall that the effective potential is a $2 \pi$-periodic function and that the frequency $\omega$ is sufficiently large.

On examining the expression (2.10) for the effective potential, the following four generic situations are found (see the graphs of the effective potential in Fig. 1):

(I) Assume that $\alpha\left|\sin \frac{\Phi}{2}\right|$ is the dominant term in the effective potential. Since it has global minima at the points $\Phi=2 k \pi, k \in \mathbb{Z}$ (see the left upper panel in Fig. 1) one expects a $2 \pi$ kink connecting two consecutive minima of
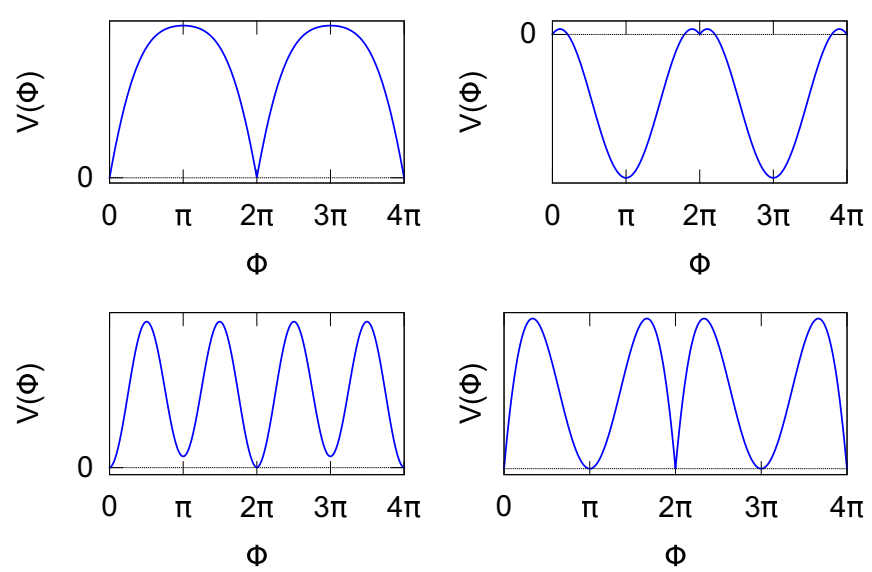

FIG. 1. (Color online) The effective potential (2.12) for the Eq. (2.1) for cases (I) (left upper panel), (II) (right upper panel), (III) (left bottom panel), and (IV) (right bottom panel). 
the potential, e.g., 0 and $2 \pi$. This case only occurs when $\alpha \gg \max \left(\left|\frac{\epsilon_{1}^{2}-\epsilon_{2}^{2}}{8 \omega^{2}}\right|,\left|\epsilon_{3}\right|\right)$.

(II) Assume that the second term $-\epsilon_{3}(1-\cos \Phi)$ is dominant. The potential therefore has its global minima at $\Phi=(2 k-1) \pi, k \in \mathbb{Z}$ (see the right upper panel in Fig. 1). Thus we expect a $2 \pi$ kink connecting two consecutive minima, e.g., $\pi$ and $3 \pi$. Obviously this is the case when $\omega$ is sufficiently large and $\epsilon_{3} \gg \alpha$ (very high-frequency regime).

(III) Consider now the case when the third term $\frac{\epsilon_{1}^{2}-\epsilon_{2}^{2}}{8 \omega^{2}}(1-$ $\cos 2 \Phi)$ in (2.10) is dominating. In this case, the effective potential has local minima at $k \pi, k \in \mathbb{Z}$ (see the left bottom panel in Fig. 1). Thus one expects two $\pi$ kinks connecting the corresponding minima. This case occurs when the frequency is such that $\frac{\epsilon_{1}^{2}-\epsilon_{2}^{2}}{8 \omega^{2}} \gg \max \left(\alpha, \epsilon_{3}\right)$, i.e.,

$$
\omega \ll \sqrt{\frac{\epsilon_{1}^{2}-\epsilon_{2}^{2}}{8 \max \left(\alpha, \epsilon_{3}\right)}} .
$$

This condition is valid, in general, for small values of $\omega$ and therefore in practice this case is forbidden.

(IV) A straightforward calculation shows that the effective potential (2.10) always has two minima in $[0,2 \pi)$. One at $\Phi=0$, which takes the value $V_{\text {eff }}(0)=0$, and the other at $\Phi=\pi$, which is equal to $V_{\text {eff }}(\pi)=\alpha-2 \epsilon_{3}$. Therefore, if we choose $\epsilon_{3} \approx \alpha / 2$, then the effective potential has a minimum at $\Phi=\pi$ with a value close to zero (see the right bottom panel in Fig. 1). Hence, one expects two $\pi$ kinks connecting the corresponding minima.

\section{B. Analysis of the model}

Let us now come back to Eq. (1.3) that models the dynamics of paramagnetic colloidal ribbons in a precessing magnetic field [5], i.e., Eq. (2.1) with the parameters (2.3).

First of all, notice that the effective potential and force (2.10) take the form

$$
\begin{aligned}
V_{\mathrm{eff}}(\Phi)= & \left|\sin \frac{\Phi}{2}\right|-2 \Delta \chi h_{+} h_{-}(1-\cos \Phi) \\
& +\frac{\Delta \chi^{2}\left(h_{+}^{2} h_{-}^{2}\right)}{2\left(\gamma^{2}+\omega^{2}\right)}(1-\cos 2 \Phi), \\
f_{\mathrm{eff}}= & \gamma(\Delta \chi)^{2} \frac{h_{+}^{4}-h_{-}^{4}}{2 \omega\left(\gamma^{2}+\omega^{2}\right)},
\end{aligned}
$$

respectively.

Our next step is to use the experimental data reported in [5] to obtain the functional dependence of $\Delta \chi$ on $\omega$.

We assume that the functional dependence of the velocity $v$ of the kinks on $\omega$ is given by a potential law $v(\omega)=a \omega^{-\delta}$, for some $\delta>0$. Using the least-squares method for the data represented in the upper panel of Fig. 4 of [5], one obtains the expression $v=314.91 / \omega^{0.58}$ with a correlation coefficient equal to 0.9197 . For the sake of simplicity in the calculations, we approximate the exponent 0.58 as $1 / 2$ or $3 / 5$. In fact, by fitting the data with the functions $V_{\text {kink }_{1}}(\omega)=$ $a \omega^{-1 / 2}$ and $V_{\text {kink }_{2}}(\omega)=a \omega^{-3 / 5}$, it is possible to describe the experimental data with a correlation coefficient 0.9163 and 0.9176 , respectively (see Fig. 2). Since these numbers (the exponents $1 / 2$ and $3 / 5$ ) are similar, in the calculations below

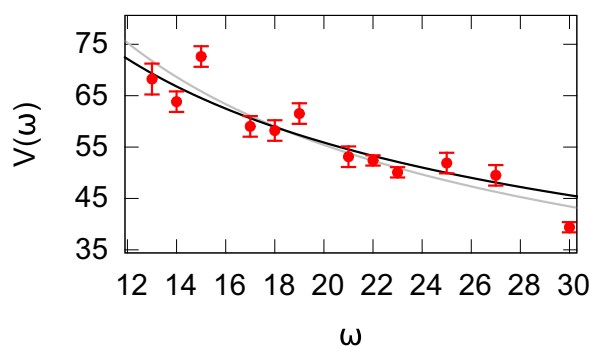

FIG. 2. (Color online) Velocity of the kink as a function of $\omega$. Dots: the experimental data; grey line: the function $V_{\text {kink }_{2}}(\omega)=$ $334.37 \omega^{-3 / 5}$; black line: $V_{\text {kink }_{1}}(\omega)=249.93 \omega^{-1 / 2}$.

we will use the simpler power law, namely

$$
V_{\text {kink }}(\omega)=\frac{a}{\sqrt{\omega}} .
$$

On the other hand, it is known [14] that the velocity of the kink solution of Eq. (2.9) (with a generic potential) is proportional to the force $f_{\text {eff }}$. Therefore, from (2.12) it follows that $V_{\text {kink }}(\omega) \sim \Delta \chi(\omega)^{2} / \omega^{3}$, from where the dependence of $\Delta \chi$ on $\omega$ is obtained:

$$
\Delta \chi(\omega)=\lambda \omega^{5 / 4},
$$

where $\lambda$ is a constant. Notice that $\Delta \chi(\omega)$ is an increasing function of $\omega$ that is small for small $\omega$. Moreover, with the choice of parameters (2.3), and taking into account that, for this problem, $\epsilon_{3}=2 \Delta \chi(\omega) h_{+} h_{-} \sim 1$ (see Table I), the condition (2.11) reads

$$
\omega \ll \frac{\sqrt{\Delta \chi(\omega) h_{+} h_{-}}}{2} .
$$

By solving the above inequality with respect to $\omega$ by using Eq. (2.14), it can be deduced that in order to have case (III), the following condition should be fulfilled:

$$
\omega \ll \omega_{\mathrm{C}}=\left(\frac{\lambda h_{+} h_{-}}{2}\right)^{4 / 3} .
$$

The above inequality means that, in general, the regime described in case (III) only holds for small values of $\omega$ and is therefore irrelevant for our purposes. Thus, we have only the following three suitable frequency regimes:

(i) Relatively low-frequency regime. If $\Delta \chi(\omega)$ is very small for relatively low frequencies, then $\left|\sin \frac{\Phi}{2}\right|$ is the dominant term in the effective potential (see the left upper panel in Fig. 1), thus one expects a $2 \pi$ kink connecting two consecutive minima of the potential, e.g., 0 and $2 \pi$. This corresponds to case (I) above.

TABLE I. Parameters of the numerical simulations of Eqs. (2.1) and (2.9). In all cases, $h_{+}=40, h_{-}=1, \gamma=4$, and $\alpha=1$. Here we use the function $\Delta \chi(\omega)$ given by Eq. (2.16).

\begin{tabular}{lcccccc}
\hline \hline Parameters $\omega$ & $\Delta \chi(\omega)$ & $\epsilon_{1}$ & $\epsilon_{2}$ & $\epsilon_{3}$ & $f_{\text {eff }}$ \\
\hline Case (i) & 15 & $4.3345 \times 10^{-3}$ & 6.939 & 6.931 & 0.346 & $2.661 \times 10^{-2}$ \\
Case (ii) & 19 & $5.824 \times 10^{-3}$ & 9.325 & 9.313 & 0.466 & $2.425 \times 10^{-2}$ \\
Case (iii) & 35 & $1.25 \times 10^{-2}$ & 20.01 & 19.99 & 1.000 & $1.841 \times 10^{-2}$ \\
\hline \hline
\end{tabular}



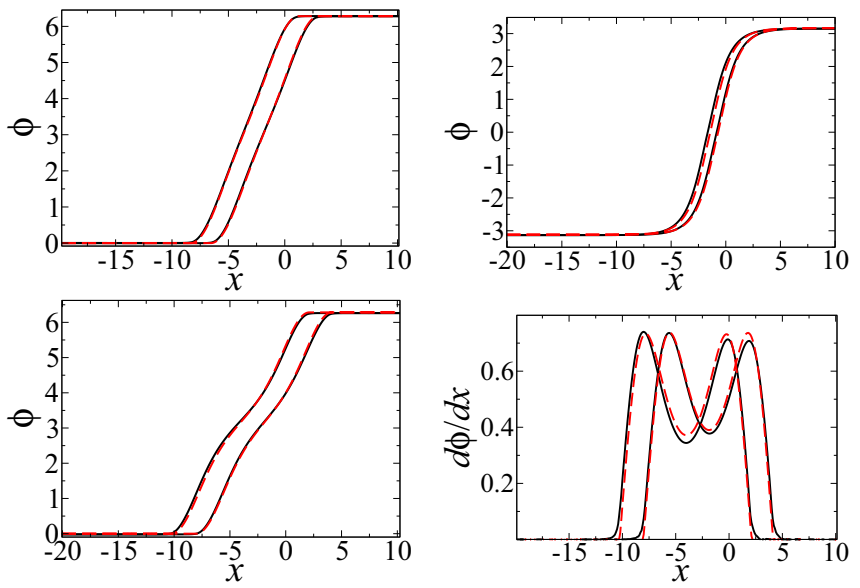

FIG. 3. (Color online) Kink profile at times $t=200$ and 400 for the three different regimes of the Eq. (2.1) (solid black lines) and the effective Eq. (2.9) (dashed red lines). The upper left panel corresponds to case (i) and shows a kink connecting $0-2 \pi$. The upper right panel corresponds to case (iii) and shows a kink connecting $-\pi-\pi$. The two plots on the bottom panel correspond to the numerical solution of the equation (left panel) and their derivatives (right panel) for case (ii) and show $\mathrm{a}-\pi-0$ and $0-\pi$ kinks. In all cases the kinks are moving to the left. The parameters are shown in Table I.

(ii) Intermediate-frequency regime. Assume that $\Delta \chi(\omega)$ increases with $\omega$ in such a way that, for an intermediate frequency,

$$
1-4 \Delta \chi h_{+} h_{-} \approx 0
$$

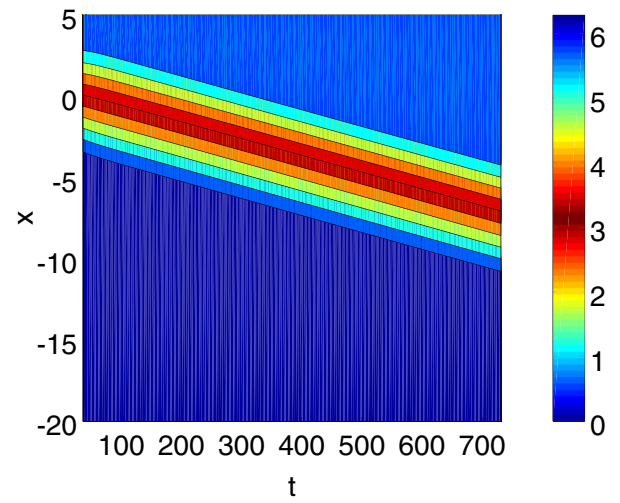

In this case [which corresponds to case (IV) above], the effective potential has a minimum at $\Phi=\pi$ with a value close to zero (see the right bottom panel in Fig. 1). One therefore expects two $\pi$ kinks connecting the corresponding minima.

(iii) Relatively high-frequency regime. If $\omega$ is sufficiently large and $2 \Delta \chi h_{+} h_{-} \gg 1$ then the second term $-2 \Delta \chi h_{+} h_{-}(1-\cos \Phi)$ is dominant (see the right upper panel in Fig. 1). Thus, we expect a $2 \pi$ kink connecting two consecutive minima, e.g., $\pi$ and $3 \pi$. This corresponds to case (II) above.

In short, we have the following picture: For a low-frequency [case (i)] $\Delta \chi \approx 0$, Eq. (2.1) exhibits a solitary wave of kink type connecting the values of the variable $\phi=0$ and $\phi=2 \pi$. By increasing $\omega, \Delta \chi$ increases until it reaches the value $\Delta \chi \approx 1 /\left(4 h_{+} h_{-}\right)$and then the kink becomes a kink-kink (or two $\pi$ kinks) connecting the values $\phi=0, \phi=\pi$, and $\phi=2 \pi$ [case (ii)]. Finally, for very large frequency [case (iii)] we recover a kink-type solitary wave which connects the values $\phi=\pi$ and $\phi=3 \pi$. All this is in agreement with the observations described in [5] and therefore Eq. (2.1) can be employed to describe the experimental results reported in [5].

\section{Numerical simulations}

In this section, we will check the theoretical predictions of the above section by providing numerical simulations of the Eq. (2.1) as well as of the effective Eq. (2.9) with the parameters (2.3).

Bellow, the constant $\lambda$ in (2.14) is fixed in such a way that, for the relatively high frequency $\omega=35, \Delta \chi(35)=0.0125$.

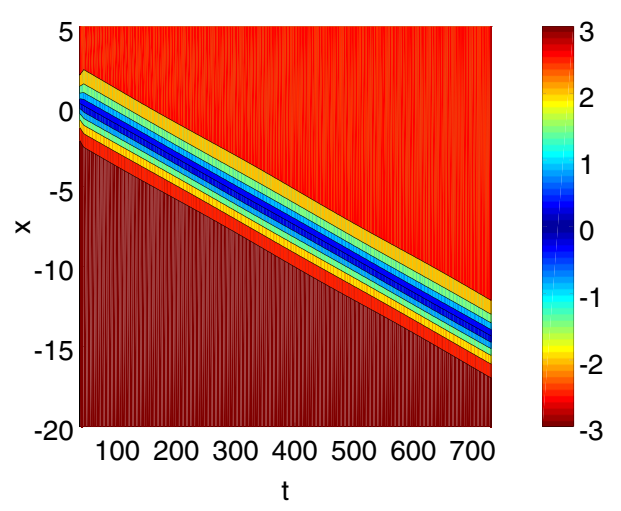

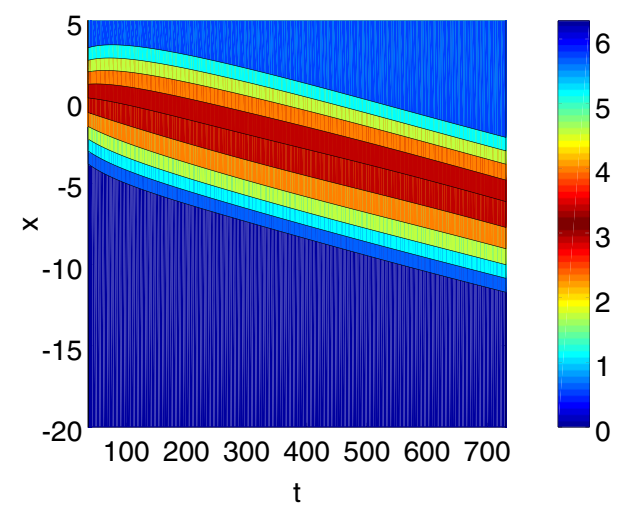

FIG. 4. (Color online) Contour plots of the kink solution for the three different regimes of Eq. (2.1). The upper left panel corresponds to case (i), the upper right panel corresponds to case (iii), and the bottom panel corresponds to case (ii) (notice a wider region around $x=0$ ). 
This yields the expression

$$
\Delta \chi(\omega)=1.468334 \times 10^{-4} \omega^{5 / 4}
$$

Using the above function, we find the value $\omega_{\mathrm{O}}$ such that $1-4 \Delta \chi(\omega) h_{+} h_{-} \approx 0$, i.e., the region corresponding to case (ii) when a kink-kink solution of the Eq. (2.1) is expected. Finally, we numerically solve the Eq. (2.1) by choosing a lower value of $\omega$ such that the potential is the same as in the left upper panel in Fig. 1.

The results of the simulations are shown in Figs. 3 and 4. In fact, in Fig. 3 we show the results of simulations of Eq. (2.1) corresponding to cases (i)-(iii) discussed in the previous section. For the derivative of the potential (2.2) we use the expression (2.4).

In all cases, we use the values $\alpha=1, f=0, h_{+}=40, h_{-}=$ 1 , and $\gamma=4, \Delta \chi(\omega)$ is given by Eq. (2.16), and the optimal frequency is $\omega_{\mathrm{O}}=20.102$. We have integrated the PDEs until time $t_{f}=400$ by using a fourth-order Runge-Kutta method with spatial discretization step $\Delta x=0.1$, time discretization step $\Delta t=0.01$, and length of the system $L=100$. For the initial condition, we use a $0-2 \pi$ kink for the low- and intermediate-frequency regimes and a $-\pi-\pi$ kink for the high-frequency regime, respectively.

In Fig. 3, we show the times $t^{*}=200$ and 400 (both are much larger than the transient time $\tau=1 / \gamma=0.25$ ). The other parameters of the simulations for each case can be seen in Table I. Notice that with the choice of parameters given in Table I, (2.15) becomes $\omega_{\mathrm{C}} \ll 4.2 \times 10^{-4}$, and therefore regime (III), as already pointed out, is forbidden. In Fig. 4, the contour plots corresponding to the three different regimes are shown.

From the graphics in Figs. 3 and 4 it is also clear that the velocity is a decreasing function of $\omega$ as was observed in the experiments.

\section{CONCLUSIONS}

In this paper we analyze Eq. (1.3) that models the dynamics of paramagnetic colloidal ribbons in a precessing magnetic field and use this analysis to explain the experimental results published previously in [5]. We have computed the shape and the dynamics of traveling twist walls in a colloidal ribbon that is dynamically self-assembled from paramagnetic colloids in water on top of a glass substrate.

From our analysis three regimes of differently shaped twist walls can be distinguished in complete agreement with the experiments reported in [5]. At low frequencies, twist walls connect lying ribbon segments with other lying ribbon segments that are twisted by $\Delta \phi_{b}=\pi(\Delta \phi=2 \pi)$. At high frequency standing ribbon segments are connected with other standing ribbon segments that are twisted by $\Delta \phi_{b}=\pi(\Delta \phi=$ $2 \pi)$. At intermediate frequencies, the twist walls are split into two walls connecting lying to standing segments that are twisted by $\Delta \phi_{b}=\pi / 2(\Delta \phi=\pi)$.
Moreover, from our study it is possible to obtain the frequency dependence of the anisotropy $\Delta \chi(\omega)$ of the magnetic susceptibility (2.16) of a ribbon of paramagnetic colloids that is subject to a dynamic external magnetic field. The increase of $\Delta \chi(\omega)$ with the modulation frequency may be due to the decrease of the fast varying function $\zeta(t)$ of the cross-section orientation at high frequencies.

\section{ACKNOWLEDGMENTS}

We acknowledge financial support through Grants No. MTM2012-36732-C03-03 (R.A.N.), No. FIS2011-24540 (N.R.Q.) from the Ministerio de Economía y Competitividad (Spain), No. FQM262 (R.A.N.), No. FQM207 (N.R.Q.), and No. FQM-7276 and No. P09-FQM-4643 (N.R.Q., R.A.N.) from Junta de Andalucía (Spain), a grant from the Alexander von Humboldt Foundation (Germany) through Research Fellowship for Experienced Researchers SPA 1146358 STP (N.R.Q.).

\section{APPENDIX: CALCULATION OF $V^{\prime}$ AND $V^{\prime \prime}$}

In Sec. II A we pointed out that the derivative of the potential $V$ is not a continuous function. Here we show how to proceed to avoid this problem. In the following we restrict ourselves to the natural open interval $I:=(-2 \pi, 2 \pi)$. Notice that the function

$$
v(\phi):=\left|\sin \left(\frac{\phi}{2}\right)\right|
$$

is not differentiable at $t=0$ in the strong sense. However, it can be approximated by a smooth function in an integral sense. Moreover, the function

$$
v^{\prime}=g(\phi):=\frac{1}{2} \operatorname{sgn}\left(\frac{\phi}{2}\right) \cos \left(\frac{\phi}{2}\right),
$$

is the weak derivative of $v$ in the usual sense of the theory of distributions. Combining this with the theory of Sobolev spaces (see, e.g., Sec. 8.2 in [15]) it can be shown that $v$ can be approximated by smooth functions $v_{n}$ in such a way that the weak derivative $v^{\prime}$ is still approximated by the derivatives $v_{n}^{\prime}$ of the approximations $v_{n}$ of $v$, being these approximations in the integral sense, i.e., in the $L^{1}$ norm. In fact, for this approximate potential (we recall that it is now a smooth function) we can write the Taylor expansion. It is clear that we can find a sequence of functions $v_{n}$ that is equal to $v$ in $I$ except for a very small neighborhood $U_{\epsilon}(0)$ of $\phi=0$ (that can be chosen arbitrarily small) and such that all the derivatives of $v_{n}$ and $v$ coincides in $I \backslash U_{\epsilon}(0)$. In particular, in this set the derivatives of $\tilde{v}$ coincides with the absolutely continuous part of the derivatives of $v$. Furthermore, if one computes $v^{\prime \prime}$ in $I$ we obtain

$$
v^{\prime \prime}=\left|\sin \left(\frac{\phi}{2}\right)\right|^{\prime \prime}=-\frac{1}{4}\left|\sin \left(\frac{\phi}{2}\right)\right|+\frac{1}{2} \delta(\phi) .
$$

Thus, the absolutely continuous part of $V^{\prime \prime}$ is $-\epsilon_{3} \cos \Phi-$ $\frac{\alpha}{4}\left|\sin \left(\frac{\Phi}{2}\right)\right|$ that is bounded by $\left|\epsilon_{3}\right|+|\alpha| / 4$. 
[1] C. A. Angell, K. L. Ngai, G. B. McKenna, P. F. McMillan, and S. W. Martin, J. Appl. Phys. 88, 3113 (2000).

[2] N. Nandi, K. Bhattacharyya, and B. Bagchi, Chem. Rev. 100, 2013 (2000).

[3] P. Guntert, C. Mumenthaler, and X. Wuthrich, J. Mol. Biol. 273, 283 (1997).

[4] N. Sreerama and R. W. Woody, Anal. Biochem. 287, 252 (2000).

[5] N. Casic, N. Quintero, R. Alvarez-Nodarse, F. G. Mertens, L. Jibuti, W. Zimmermann, and T. M. Fischer, Phys. Rev. Lett. 110, 168302 (2013).

[6] I. M. Gel'fand and G. E. Shilov, Generalized Functions: Properties and Operations (Academic, New York, 1964).

[7] Yu. S. Kivshar, N. Gronbech-Jensen, and M. R. Samuelsen, Phys. Rev. B 45, 7789 (1992).
[8] Yu. S. Kivshar, A. Sánchez, and L. Vázquez, Phys. Rev. A 45, 1207 (1992).

[9] L. D. Landau and M. Lifshitz, Mechanics (Pergamon, Oxford, 1960).

[10] Yu. S. Kivshar, N. Gronbech-Jensen, and R. D. Parmentier, Phys. Rev. E 49, 4542 (1994).

[11] Yu. S. Kivshar and K. H. Spatschek, Chaos Solitons Fractals 5, 2551 (1995).

[12] V. Zharnitsky and D. Pelinovky, Chaos 15, 037105 (2005).

[13] C. A. Condat, R. A. Guyer, and M. D. Miller, Phys. Rev. B 27, 474 (1983).

[14] D. W. McLaughlin and A. C. Scott, Phys. Rev. A 18, 1652 (1978).

[15] H. Brezis, Functional Analysis, Sobolev Spaces and Partial Differential Equations (Springer, New York, 2010). 